\title{
Numerical Investigation of Supersonic Channel Flow with Oscillatory Backpressures
}

\author{
Peizhao Sun, Nansheng Liu, Jiming Yang and Xiyun Lu* \\ Department of Modern Mechanics, University of Science and Technology of China, \\ Hefei 230026, Anhui, China
}

Received 1 April 2018; Accepted (in revised version) 22 May 2018

\begin{abstract}
The investigation of supersonic channel flow with periodic oscillatory backpressures at the outlet of the channel was performed using large-eddy simulation for the inlet free-stream Mach number 4 and the Reynolds number approximately $5.2 \times 10^{4}$ based on the height of the channel. Results have been validated carefully against our experimental data. Three typical backpressures are considered for constant backpressure and both periodic oscillatory backpressures with low and high frequency. The oscillatory backpressure can obviously influence the flow features occurring up to the middle region of the channel for the low frequency case and the downstream region for the high frequency case. Obvious differences of phase-averaged quantities at different phases are observed for the low frequency backpressure while the differences are relatively small for the high frequency backpressure. The spectral analysis reveals that the flow field experiences a periodic-like evolution of flow structures including shocks and vortices for the low frequency backpressure, resulting in the enhancement of turbulence fluctuations due to the complicated interaction of shocks and vortices.
\end{abstract}

AMS subject classifications: 76F65, 76L05, 76F70

Key words: Large eddy simulation, shock wave, turbulent flow.

\section{Introduction}

The supersonic air breathing propulsion system includes the inlet and the isolator which are the precombustion compression components [1,2]. In the inlet, the incoming flow is compressed through a series of shocks before entering the combustion chamber. To isolate the effect of flight conditions on the precombustion shock structure, a nearly parallel walled duct named isolator is placed between the inlet and the combustor [3-5]. The isolator contains a time-varying shock train system providing a stable flow to the

${ }^{*}$ Corresponding author.

Emails: pzsun@mail.ustc.edu.cn (P. Z. Sun), lns@ustc.edu.cn (N. S. Liu), jmyang@ustc.edu.cn (J. M. Yang), xlu@ustc.edu.cn (X. Y. Lu) 
combustion process and is highly susceptible to the flow induced instabilities. Hence, the pressure change caused by the combustion will lead to complicated flow phenomena and mechanisms in the isolator [6].

The unsteady properties of the internal flow caused by high backpressure were investigated. Wagner et al. [7] dealt with the dynamics of internal flow influenced by the backpressure generated by a deflecting flap at the downstream end of the isolator by means of the PIV measurement and observed that the unsteadiness of the internal flow is associated with boundary layer separation. Tan et al. [8] experimentally investigated the unstart flows of a rectangular hypersonic inlet at a freestream Mach number of 5 and demonstrated the typical external/internal flow patterns and the unsteady behaviors of surface pressures. Recently, Li et al. [6] designed a two-dimensional hypersonic inlet/isolator model to investigate the complicated flow structures experimentally. They found that the upstream-propagating shocks in the isolator play an important role on the flow properties and the formations of the upstream-propagating shocks are related to the downstream-propagating compression waves/shock waves that encounter the throttling section.

On the other hand, some numerical simulations were carried out to investigate the flow behaviors in the inlet-isolator system. Koo et al. [5] performed a large-eddy simulation (LES) of the Mach 5 inlet-isolator system adopted by the experiment [7]. Two cases including a started isolator and transient unstart propagation were considered to analyze the characteristics of flow separation and shock waves. It is demonstrated that the LES technique is able to predict fully started flow accurately and to capture the largescale features of the unstart process. Ingenito et al. [9] also performed the LES of the HyShot combustor system to analyze the effects of mixing and combustion. Cocks et al. [10] carried out a detached-eddy simulation (DES) of supersonic combustor to study the characteristics of the cavity flameholder and identified that the prescription of inflow turbulence plays an important role on the accurate prediction of the shock train. $\mathrm{Kr}-$ ishnan et al. [11] also performed the LES of Mach 8 inlet system to analyze the transition mechanisms on the compression ramps and the statistical characteristics of the flow field.

Actually, the backpressure caused by the combustion downstream has remarkable variation. Although the unsteadiness of the internal flow in an isolator has been widely investigated, the situations with periodic oscillatory backpressure are rarely studied. Bur et al. [12] performed an experiment on the periodic motions of shocks in transonic channel flow, where the motion was generated by the rotating elliptical or rectangular shaft at the exit of the channel. The influence of the rotating shaft shape on the shock oscillation in the channel was investigated. Based on the experimental measurement, the numerical simulation by means of the two-dimensional unsteady Reynolds-averaged Navier-Stokes (RANS) solution was also done. Further, Bruce and Babinsky [13] experimentally investigated an oscillating normal shock wave subjected to unsteady periodic forcing in a duct, where the periodic backpressure was generated by an elliptical cam placed in the downstream of the duct. The interaction between the moving shock and the boundary layer was analyzed and an inviscid analytical model was proposed to reproduce the dynamics 
of the unsteady shock motion. Recently, Cheng et al. [14] carried out an experimental study on the unsteady features of the oblique shock train in a Mach 2.7 flow and noted that the oblique shock train undergoes a periodic oscillation motion in the streamwise direction with the periodic downstream pressure perturbations.

Moreover, some numerical simulations on the internal flow with time-dependent backpressure were carried out. Su et al. [15] investigated the effects of periodic backpressure on the flow field unsteadiness in an internal flow and identified that the frequency and amplitude of backpressure have an important influence on the pressure distribution in the flow field. Xu et al. [16] simulated the oblique shock train using Fluent and proposed the relationship between the pressure variation and the sudden sharp forward movement of the shock train. Trapier et al. [17] investigated the supersonic inlet buzz in a rectangular inlet with Mach 1.8 by means of DES. Moreover, the internal flow influenced by high backpressure can induce complicated flow behaviors including significant time-varying flow evolutions and turbulent features, which also have an important influence on aerodynamic performance and safety of the flight $[7,18]$. However, the physical mechanisms dictating the complicated phenomena are still fully unclear and are of great interest for further detailed studies.

In the present study, the isolator is simplified into a supersonic channel. The statistical properties of the internal flow inside supersonic channel with periodic oscillatory backpressure are investigated by means of LES for the inlet free-stream Mach number 4 and the Reynolds number approximately $5.2 \times 10^{4}$ based on the height of the channel. The characteristics of flow structures and statistical turbulence features caused by the periodic backpressure is investigated for a better understanding of the underlying mechanisms.

This paper is organized as follows. The mathematical formulation, numerical method, computational overview and validation are presented in Section 2. Detailed results and discussion are then given in Section 3 and the concluding remarks are finally addressed in Section 4.

\section{Computational strategy}

\subsection{Mathematical formulation and numerical method}

We here simplified the isolator of our previous experiment [6] as a supersonic turbulent channel flow with its configuration schematic shown in Fig. 1. To investigate the supersonic turbulent channel flow, the three-dimensional Favre-filtered compressible NavierStokes equations in generalized coordinates are employed. The equation of state for an ideal gas is used and the molecular viscosity is assumed to obey the Sutherland law. To non-dimensionalize the equations, the free stream variables including the density $\rho_{\infty}$, the temperature $T_{\infty}$ and the speed of sound $a_{\infty}$, and the height of the channel $h$ are used. The LES is implemented in the present work for turbulence closure. Some terms in the Favrefiltered equations arise from unsolved scales and need to be modeled in terms of resolved scales. Then, dynamic subgrid-scale models for compressible flows are employed. A de- 


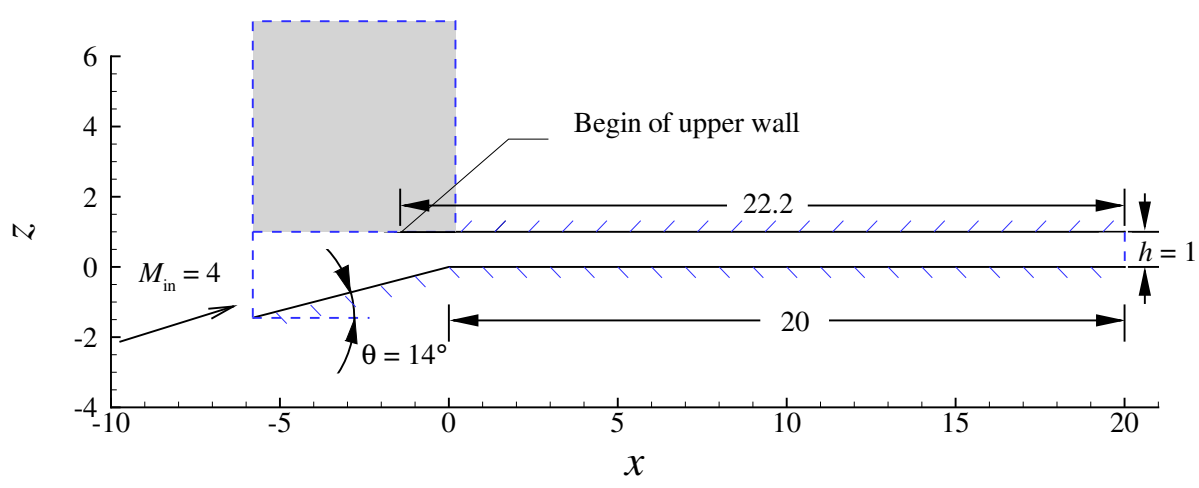

Figure 1: Schematic of the channel model for a simplified isolator.

tailed description of the mathematical formulation of the non-dimensionalized equations and the subgrid-scale models has been given in our previous papers [19-21].

The inflow boundary condition is treated using a synthesized approach $[22,23]$ which has been extensively tested in previous literature [23-26]. The velocity disturbances at the inlet are determined and the random velocity fluctuations with a maximum amplitude $4 \%$ of the free-stream velocity are added to the velocity components according to previous studies [23-27].

\subsection{Computational overview and validation}

As shown in Fig. 1, the inlet free-stream Mach number is $M_{i n}=4$, the total pressure and total temperature at the inflow condition are $P_{0}=1.27 \mathrm{MPa}$ and $T_{0}=810 \mathrm{~K}$, respectively, and the Reynolds number based on the height of the channel is $5.2 \times 10^{4}$. In addition, we have performed the experimental investigation for the configuration and parameters described above [6], which can be used to validate the present calculation.

In this study, the flow domain is discretized by equally spaced grids along the streamwise $(x)$ and spanwise $(y)$ directions and a stretched grid in the wall-normal direction $(z)$, so that there are enough points near the two walls to capture the boundary layer information. Based on careful examination, the computational domain is used as $-6 \leq x \leq 20$ in the streamwise direction and $-1.25 \leq y \leq 1.25$ in the spanwise direction, where the coordinates $x, y$ and $z$ are non-dimensionalized by the height of the channel. A ramp with an angle of $14^{\circ}$ is placed before the lower wall which ends at $x=0$. The periodic condition is applied in the spanwise direction. The no-slip and adiabatic wall conditions are applied on the walls. In addition, an inlet region exhibited by the shaded area in Fig. 1 is used to simulate the inlet flow, and its computational domain covers $-6 \leq x \leq 0$ in the streamwise direction, $-1.25 \leq y \leq 1.25$ in the spanwise direction and $1 \leq z \leq 7$ in the normal direction. The details of the grid resolution for the computational domain are shown in Table 1.

The backpressure is added at the outlet of the channel and three typical cases are considered for constant backpressure and both oscillatory backpressures with low and high 
Table 1: Details of grid resolution for computational domain.

\begin{tabular}{||c|c|c|c|c||}
\hline Case & $\begin{array}{c}\text { Channel region } \\
\left(N_{x} \times N_{y} \times N_{z}\right)\end{array}$ & $\begin{array}{c}\text { Inlet region } \\
\left(N_{x} \times N_{y} \times N_{z}\right)\end{array}$ & $a_{\infty} \Delta t / D$ & $\Delta z^{+}$ \\
\hline Grid 1 & $951 \times 81 \times 201$ & $501 \times 81 \times 201$ & 0.0005 & $<1$ \\
Grid 2 & $801 \times 71 \times 161$ & $301 \times 71 \times 161$ & 0.0005 & $<1$ \\
Grid 3 & $601 \times 51 \times 121$ & $251 \times 51 \times 121$ & 0.001 & $<1$ \\
\hline
\end{tabular}

frequency. The constant backpressure is prescribed as $140 p_{\infty}$ and the periodic oscillatory backpressure is presented as

$$
p_{b}=\bar{p}+\Delta p \sin \left(2 \pi k_{s} t\right),
$$

where $\bar{p}=140 p_{\infty}, \Delta p$ is the amplitude of the pressure oscillation, which is chosen as $5 p_{\infty}$, and $k_{S}$ is the Strouhal number. Furthermore, the physical frequency is chosen as $\tilde{f}_{L}=200 \mathrm{~Hz}$ with Strouhal number $S t_{L}=k_{S}=\tilde{f}_{L} h / a_{\infty}=0.01$ for low frequency backpressure case and $\tilde{f_{H}}=2000 \mathrm{~Hz}$ with $S t_{H}=k_{S}=0.1$ for high frequency case based on some experiments $[6,28,29]$. Due to the oscillatory backpressure, the phase $T / 4$ with $T$ being the oscillatory period experiences the backpressure peak with its value $145 p_{\infty}$, and the phase $3 T / 4$ experiences the valley with $135 p_{\infty}$.

Some symbols used in this paper are introduced as follows: $\langle\phi\rangle$ means the average in time and in the spanwise direction, and $\{\phi\}=\langle\rho \phi\rangle /\langle\rho\rangle$ with a variable $\phi$. Then, the fluctuations are obtained as $\phi^{\prime}=\phi-\langle\phi\rangle$ and $\phi^{\prime \prime}=\phi-\{\phi\}$, respectively.

Assessment of grid resolution on calculated results has been carried out. Fig. 2(a) shows the distributions of the pressure fluctuation $p_{r m s}^{\prime}$ on the upper wall for constant backpressure $140 p_{\infty}$, which are calculated by different grid resolutions in Table 1 . It is identified that the results for grids 1 and 2 nearly collapse together with the increase of grid resolution, indicating a reasonable convergence for the grid resolutions. To make the prediction accurate, the results given below were calculated using the finest grid resolution of grid 1.

Moreover, a comparison between numerical result and experimental data [6] is performed. Fig. 2(b) shows the distribution of the mean pressure averaged in time and the spanwise direction. The calculated result without the backpressure agrees reasonably with the experimental data [6]. Meanwhile, the distributions of the mean pressure with constant backpressures $135 p_{\infty}$ and $140 p_{\infty}$ are also shown. The pressure distributions with constant backpressures are similar to that without the backpressure in the upstream region of the channel and then exhibit a significant increase in the downstream region. Moreover, the pressure increase caused by the backpressure $135 p_{\infty}$ reasonably locates further downstream of the channel compared to the pressure increase location induced by the backpressure $140 p_{\infty}$.

We further analyze the appropriateness of the grid resolution used in this study in terms of the Kolmogorov scale. The Kolmogorov scale $\eta$ is estimated as $\eta / h \sim \mathcal{O}\left(10^{-2}\right)$ and the grid spacing is $\delta / h \sim \mathcal{O}\left(10^{-2}\right)$. Then, the ratio is $\delta / \eta \sim \mathcal{O}(1)$. Usually $\delta / \eta \sim$ 

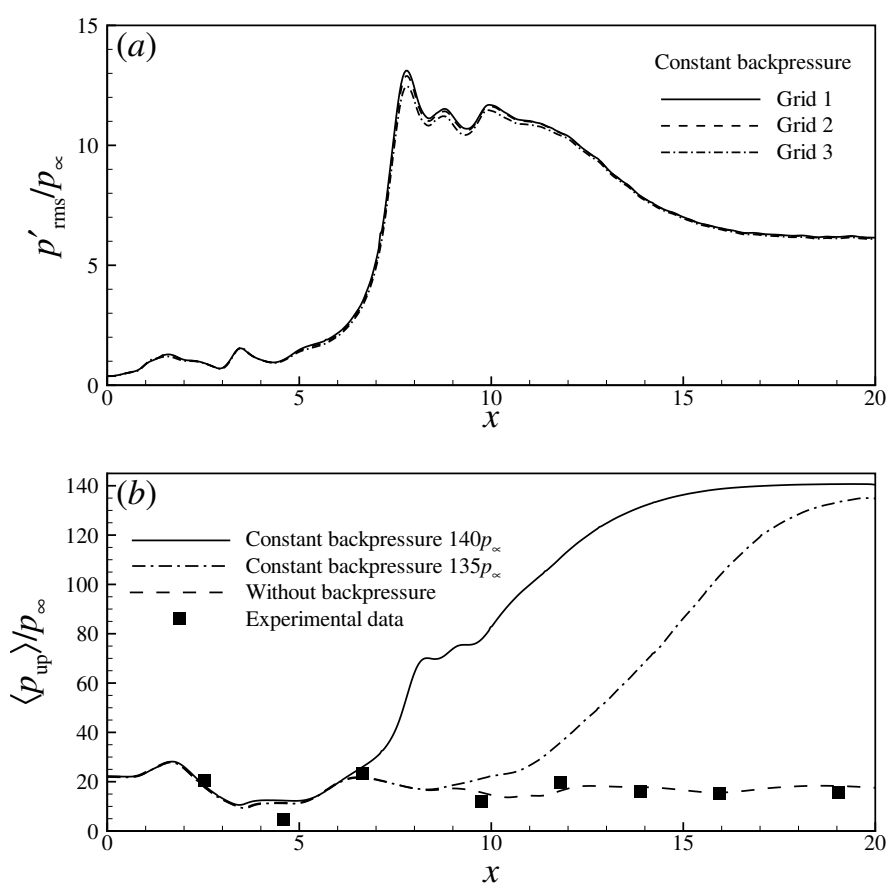

Figure 2: Validation of the present calculation: (a) pressure fluctuation $p_{r m s}^{\prime} / p_{\infty}$ on the upper wall calculated by different grid resolutions, (b) comparison of the mean pressure on the upper wall $\left\langle p_{u p}\right\rangle / p_{\infty}$ between the calculated results and experimental data [6] without the backpressure. The profiles of the mean pressure on the upper wall $\left\langle p_{u p}\right\rangle / p_{\infty}$ with backpressures $135 p_{\infty}$ and $140 p_{\infty}$ are also plotted for comparison, where the mean pressure $\langle p\rangle$ is calculated by the average in time and the spanwise direction.

$\mathcal{O}\left(10^{2}\right)$ in the LES of turbulent flow [30], thus the grid resolution used here is more sufficient to resolve turbulence. In addition, the present numerical strategy has already been applied with success to a wide range of turbulent flows, such as compressible flows over a wavy cylinder [20] and past an aerofoil [31], and a jet from a blunt body opposing a supersonic flow [21].

\section{Results and discussion}

\subsection{Flow field statement}

\subsubsection{Instantaneous flow field}

We first discuss the influence of the backpressures on the flow structures based on the instantaneous flow fields in Fig. 3. As shown in Fig. 3(a) for the flow structure with the constant backpressure in Eq. (2.1), the main features of flow field consist of a series of shock waves which are reflected between the two walls in the upstream region of the channel. The incident shock (IS) caused by the upper wall impinges near the end 

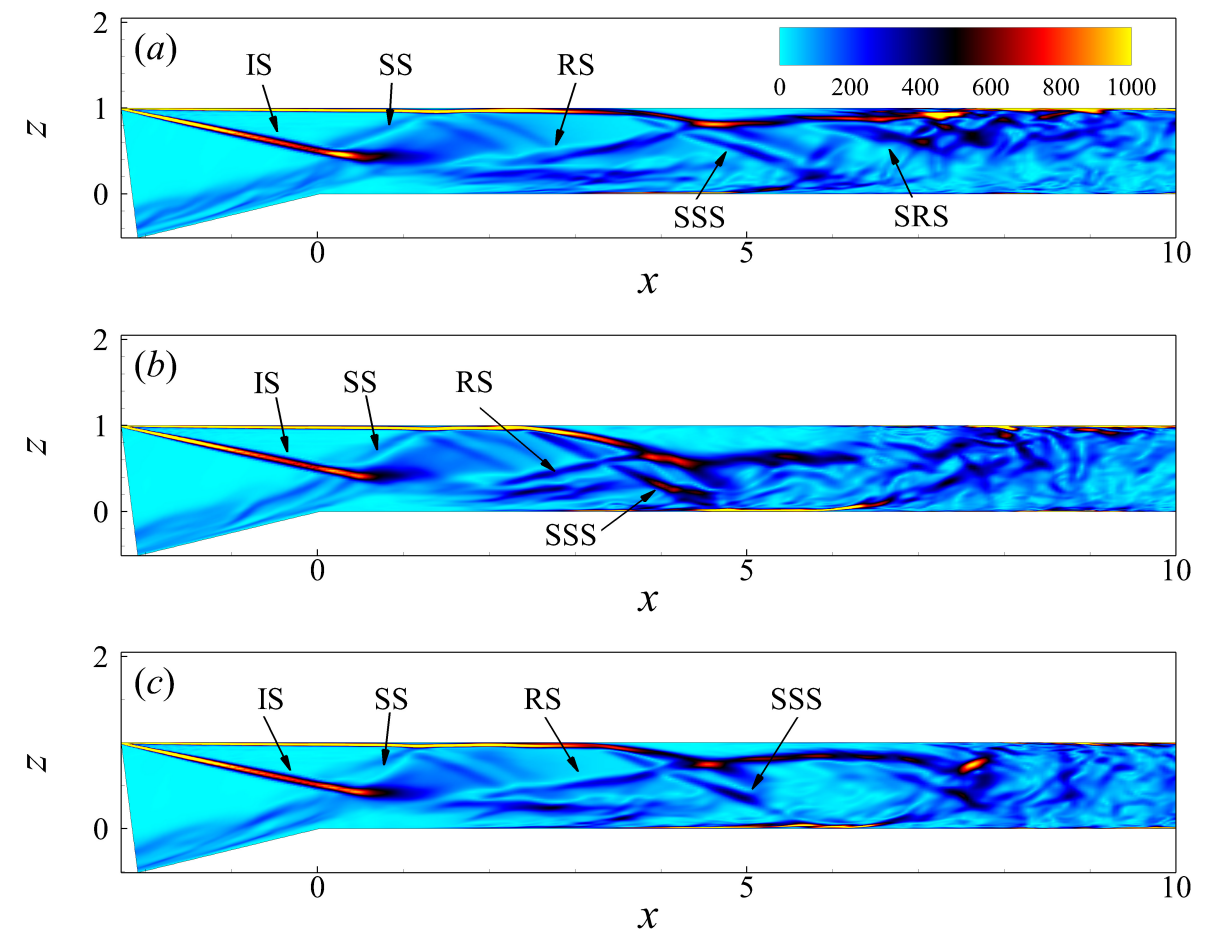

Figure 3: Numerical schlieren-like visualizations by contours of $\|\nabla \bar{\rho}\|$ in the $x-z$ plane for (a) the constant backpressure, (b) the low frequency backpressure and (c) the high frequency backpressure at the phase T/4. The label IS represents the incident shock, SS the separation shock, RS the reflected shock, SSS the secondary separation shock, and SRS the secondary reflected shock.

of the ramp and induces a local flow separation. Then the induced separation shock (SS) and the reflected shock (RS) impact on the upper wall. A small separation bubble is generated by the RS, which further causes a secondary separation shock (SSS) and a secondary reflected shock (SRS). The SRS is somewhat not obvious due to the influence of the backpressure, while the relevant influence on the flow structures in the upstream region of the channel $(x<6)$ is weak, which means the backpressure capability in the channel $[6,32]$. The location of the flow separation caused by the backpressure is related to the impingement location of the shock. Hence, there exist complicated flow structures in the region of $x<10$, including shock waves, flow separations and shear layers. In the downstream region of $x>10$, the shock waves almost disappear and the flow properties are dominated by fully developed turbulence.

Figs. 3(b) and (c) show the instantaneous flow fields for the low and high frequency backpressures at the phase $T / 4$, respectively. The low frequency backpressure induces a serious adverse pressure gradient behind the SSS in Fig. 3(b), which results in the further upstream location of the SSS, the disappearing of the SRS and the enhancement of the flow separation on the upper wall. The similar features are also observed for the high frequency backpressure in Fig. 3(c). The flow separation on the upper wall becomes 
weak and experiences a reattachment. The SRS still appears under the influence of the high frequency backpressure. In addition, the existence of the oscillatory backpressure also enhances the unsteadiness of the flow separation.

\subsubsection{Mean pressure and local Mach number}

The distributions of mean pressure and local Mach number along the centerline are shown in Fig. 4, where the mean value is calculated by the average in time and the spanwise direction. As shown in Fig. 4(a), the distributions of the mean pressure for the three cases coincide with each other for $x<3$ because of weak backpressure capability. As the oscillatory amplitude of the SSS increases under the influence of low frequency backpressure, the distribution exhibits a change for $3<x<7$. Besides, the mean pressure for the other two cases exhibits a tendency of first increasing and then decreasing for $3<x<7$, and achieves a peak value at $x=5$ due to the oscillation of the SSS. As the existence of the SRS, the mean pressure has a quick increase to the peak for the constant and high frequency backpressure around $x=7$, which exhibits an increase for the low frequency backpressure.

To understand the flow feature related to the shock/turbulence interaction, the profiles of mean local Mach number along the centerline are plotted in Fig. 4(b). The distributions of the local Mach number clearly distinguish the supersonic and subsonic regions. For $x<10$, the oblique shock wave system exists in the channel, which exhibits a supersonic region. While in the downstream of the channel, the flow is decelerated across the shocks and the turbulence is enhanced due to the unsteadiness of separated shear layers. As the influence of the low frequency backpressure, the flow separation zone on the upper wall becomes large and the SSS experiences a large range oscillation, which results in a change of the Mach number for $3<x<7$. Because of the slight oscillation of the SSS in the other two cases, the profiles exhibit somewhat smaller distribution. Further, the disappearance of the SRS for the low frequency backpressure leads to a gradual decrease of the Mach number when $x>7$, while a quick decrease is observed for the other two
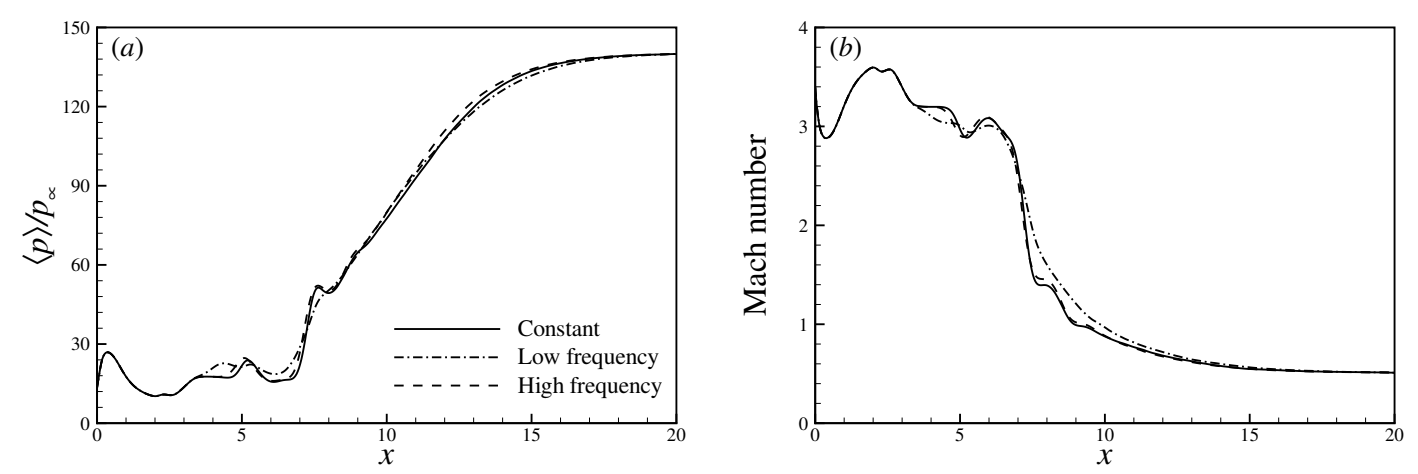

Figure 4: Distributions of $(a)$ the mean pressure and (b) the mean Mach number along the centerline of the channel. 
cases.

\subsubsection{Phase-averaged flow field}

In order to investigate the flow behaviors at different phases of oscillatory backpressure, Fig. 5 shows the profiles of phase-averaged sonic line. It is observed from Fig. 5(a) that there exists the change of sonic line during one cycle due to the influence of oscillatory backpressure. At the phases $T / 4$ and $3 T / 4$, the flow separation region on the upper wall is smaller than that at $2 T / 4$. The supersonic region is the longest at $4 T / 4$ and the shortest at $2 T / 4$. For comparison, the profiles of phase-averaged sonic line for the high frequency backpressure are also shown in Fig. 5(b). The profiles have a little difference among several phases, indicating that there exists weak influence on the flow structure subject to the high frequency backpressure.

Fig. 6 shows the distributions of the phase-averaged pressure along the centerline. The profiles at different phases demonstrate obvious difference for the low frequency backpressure in Fig. 6(a). The curves at $T / 4$ and $3 T / 4$ are nearly coincident with the mean pressure $\langle p\rangle$ for the constant backpressure, while obvious differences occur at $2 T / 4$ and $4 T / 4$. The profile at $2 T / 4$ has a peak around $x=4$, which is related to the forward motion of the SSS caused by the flow separation on the upper wall. Meanwhile, the strong separation also leads to large oscillation of the SSS, which further induces a pressure rise. Thus the curve at $2 T / 4$ exhibits a quick increase around $x=6$ due to the flow separation zone increase on the lower wall. At $4 T / 4$, the profile is lower than that for the constant backpressure in the region $4<x<7$, which corresponds to the flow separation decrease on the lower wall. Moreover, the pressure at $4 T / 4$ has a peak around $x=5$ which is associated with the downstream motion of the SSS.

Fig. 6(b) also shows the profiles of the phase-averaged pressure for the high frequency backpressure. It is seen that the distributions at various phases exhibit somewhat small
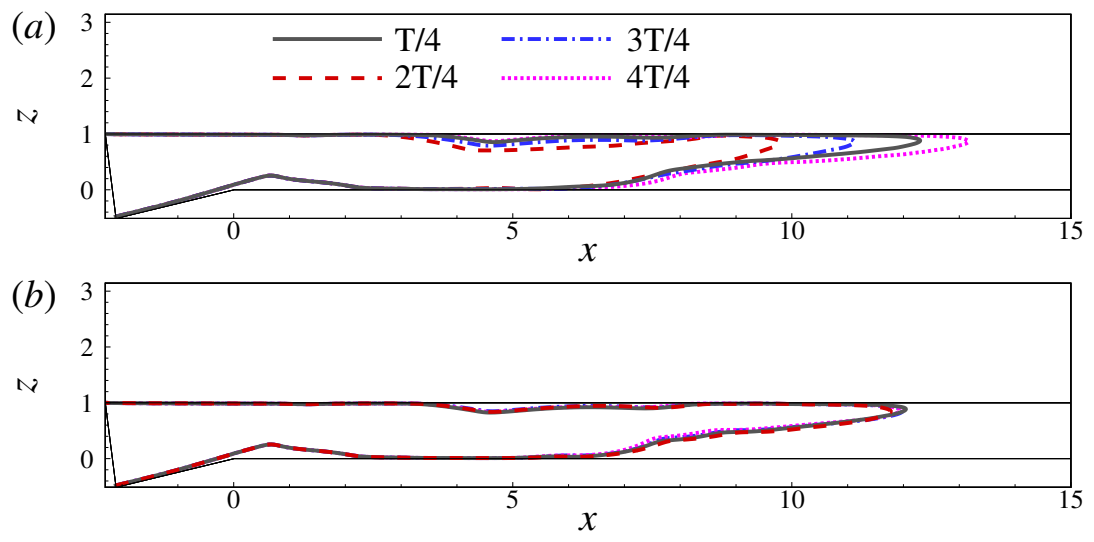

Figure 5: The distributions of the phase-averaged sonic lines in the $x-z$ plane for (a) the low frequency backpressure and (b) the high frequency backpressure. 

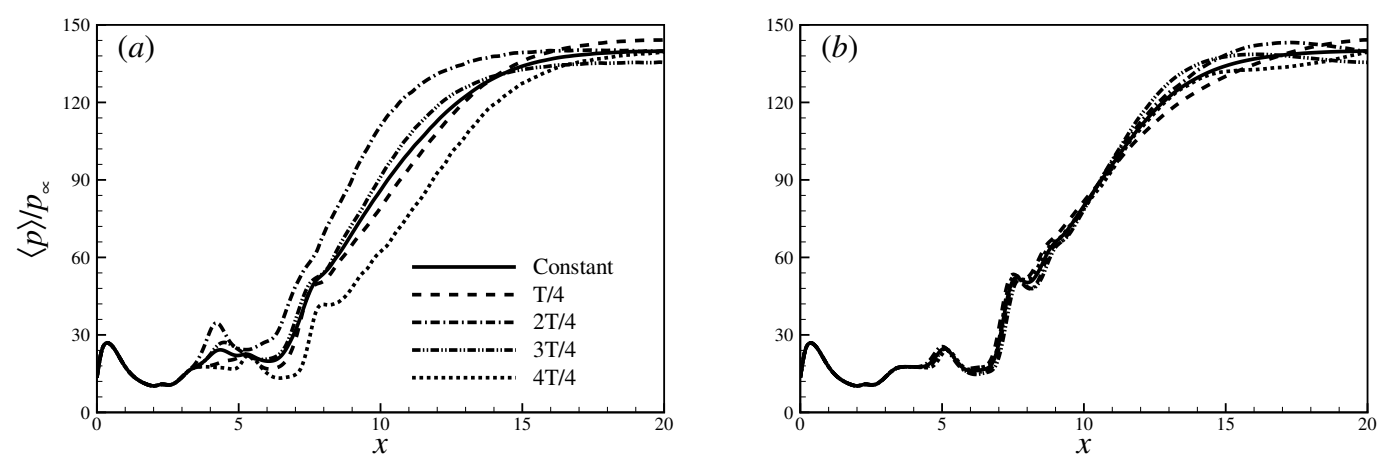

Figure 6: Streamwise distributions of the phase-averaged pressure along the centerline for (a) the low frequency backpressure and (b) the high frequency backpressure. The solid line denotes the mean pressure $\langle p\rangle$ averaged in time and spanwise direction for the case with constant backpressure.

difference. The unsteadiness of flow structure in the region of $6<x<10$ results in small deviations of the phase-averaged pressure from the mean pressure $\langle p\rangle$. Moreover, an obvious periodic-like variation is observed in the downstream for $x>12$ induced by the periodic backpressure. This feature indicates that the high frequency backpressure mainly influences the flow structure in the downstream region of the channel.

The correlation coefficient of the pressure fluctuation $p^{\prime}$ determined at a reference location $x_{0}$ is discussed, which is calculated by [33]

$$
R_{p}(x)=\frac{\left\langle p^{\prime}\left(x_{0}\right) p^{\prime}(x)\right\rangle}{\left\langle p^{\prime}\left(x_{0}\right)\right\rangle^{1 / 2}\left\langle p^{\prime}(x)\right\rangle^{1 / 2}}
$$

where $x$ is the streamwise coordinate along the centerline. Fig. 7 shows the distribution of the correlation coefficient of $p^{\prime}$ along the centerline. Two typical reference locations are selected. One locates at $x_{0}=3.85$ near the SSS, and the other at $x_{0}=19.8$ close to the outlet of the channel. As shown in Fig. 7(a), the correlation coefficient exhibits a peak at $x=3.85$ for the constant backpressure and approaches zero in the downstream of the channel, indicating a weak relationship of the fluctuations in the upstream and downstream of the channel. Moreover, the profile of the correlation coefficient for the high frequency backpressure exhibits the similar behavior to the constant backpressure case. For the low frequency backpressure, the profile also reaches another peak value around $x=6$ except for the peak at $x_{0}=3.85$, which is associated with the oscillation of the reflected shock of SSS. Then, the correlation decreases gradually to a negative distribution near the outlet of the channel, which is related to the phase difference between the backpressure and the oscillation of SSS.

It is observed from Fig. 7(b) that the peak of correlation coefficient at $x_{0}=19.8$ decreases quickly for the constant backpressure, indicating that the oscillatory backpressure can enhance the correlation in the downstream of the channel. The correlation is nearly zero in the whole flow field for the constant backpressure. Moreover, the correlation for 

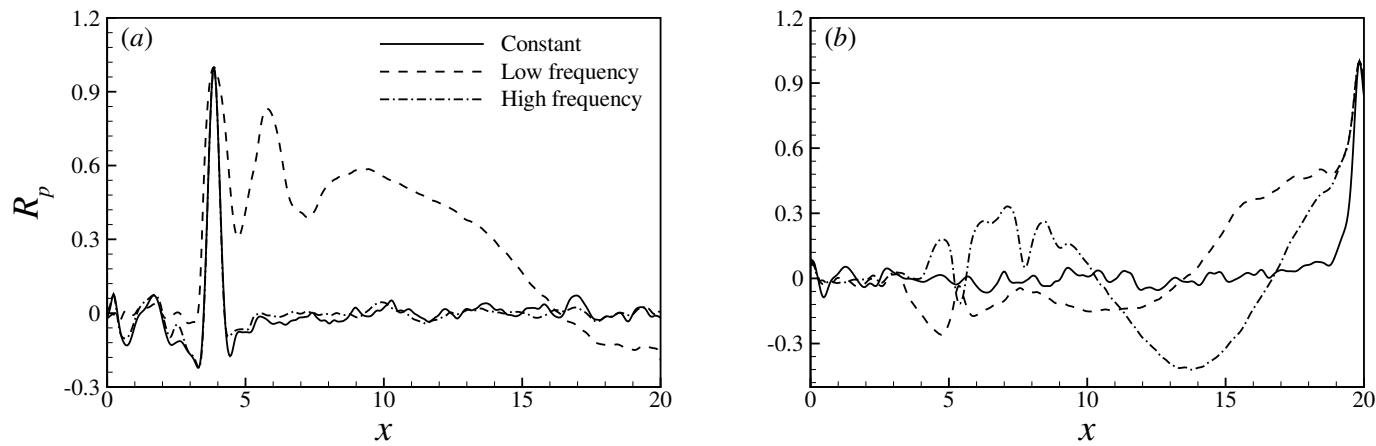

Figure 7: Distributions of the correlation coefficient of pressure fluctuation between $x_{0}$ and various positions along the centerline for (a) $x_{0}=3.85$ and (b) $x_{0}=19.8$.

the low frequency backpressure exhibits higher value distribution when $x>15$ and gradually decreases in the upstream region. The negative correlation also occurs for $3<x<13$ due to the phase change between the forward and backward motion of flow structure and the oscillatory backpressure. Further, the correlation for the high frequency backpressure has a remarkable negative distribution around $x=14$, indicating that the influence of the oscillatory backpressure on the flow structures mainly appears in the downstream region of the channel. The correlation for $4<x<10$ exhibits an obvious variation which is related to the high frequency oscillation of flow structure.

\subsection{Turbulent statistical features}

\subsubsection{Turbulent kinetic energy}

From the preceding analysis on the shock waves, flow separations and their complicated interactions, these intricate flow phenomena have a significant influence on the turbulent fluctuations. To assess the turbulent features, the specific turbulent kinetic energy (TKE) defined as $k=\left\{u^{\prime \prime}{ }_{i} u^{\prime \prime}{ }_{i}\right\} / 2 U_{\infty}^{2}$ is investigated in terms of the phase-averaged quantities.

The vertical distributions of the phase-averaged TKE at several streamwise locations are shown in Fig. 8 for low frequency backpressure. From Fig. 8(a) at $x=1.75$, the curves at different phases nearly collapse together, indicating that the disturbances from the oscillatory backpressure have little influence on the flow behaviors. There exist two peaks near the walls, which are associated with the local flow separations on the walls. Then, from Fig. 8(b) at $x=5$, as a result of the oscillation of the SSS induced by the strong flow separation on the upper wall, the distributions at different phases exhibit significant discrepancies. Two peaks are also observed near the walls, while the peak value near the upper wall becomes higher due to the flow separation on the upper wall. Then, the more severe flow separation occurs on the lower wall at $x=7.5$ and the peak near the lower wall dominates the TKE again as shown in Fig. 8(c). Meanwhile, compared to the positions $x=1.75$ and 5 , the phase-averaged TKE exhibits an increase near the centerline because 

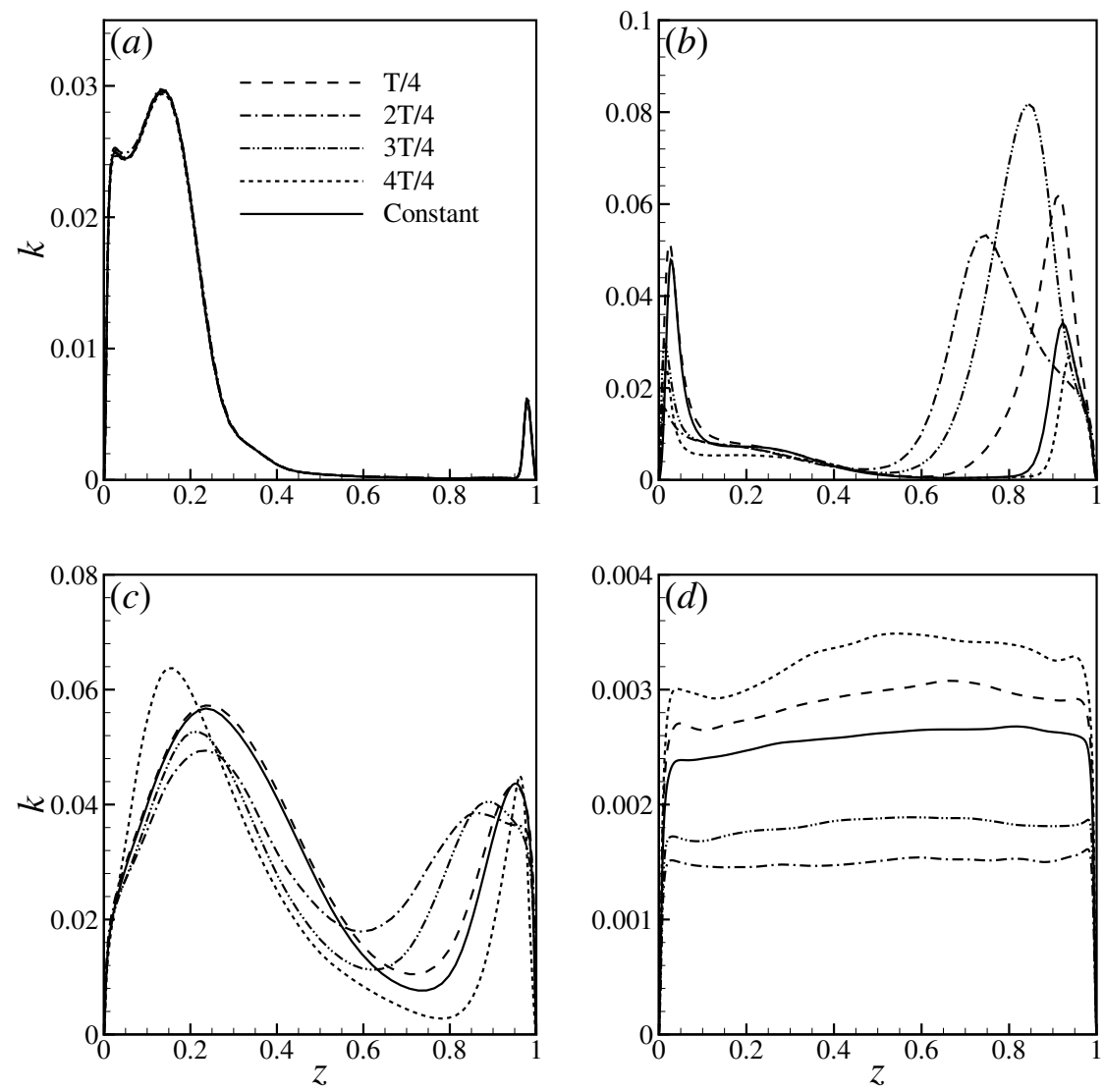

Figure 8: The vertical distributions of the phase-averaged TKE for low frequency backpressure at several streamwise locations $x=$ (a) 1.75 , (b) 5 , (c) 7.5 and (d) 18 , respectively. The solid line denotes the TKE for the constant backpressure.

of turbulence developing in the core region of the channel. Furthermore, fully developed turbulence dominates flow features in the downstream of the channel in Fig. 8(d) and the TKE exhibits gentle variation in the channel except for the near-wall regions. The variation of the TKE distributions is obviously influenced by the oscillatory backpressure in the downstream of the channel.

Fig. 9 shows the vertical distributions of the phase-averaged TKE for the high frequency backpressure. The distributions in Fig. 9(a) at $x=1.75$ match with those in Fig. 8(a). Then, from the profiles in Fig. 9(b) at $x=5$, the TKE exhibits a lower peak value near the upper wall in comparison with that near the lower wall, which is related to the local flow separations near the walls. In the downstream, the profiles in Figs. 9(c) and (d) have the similar behavior to the counterparts in Figs. 8(c) and (d). Essentially, the distributions at several phases exhibit relatively small difference among them compared to the low frequency backpressure in Fig. 8. 

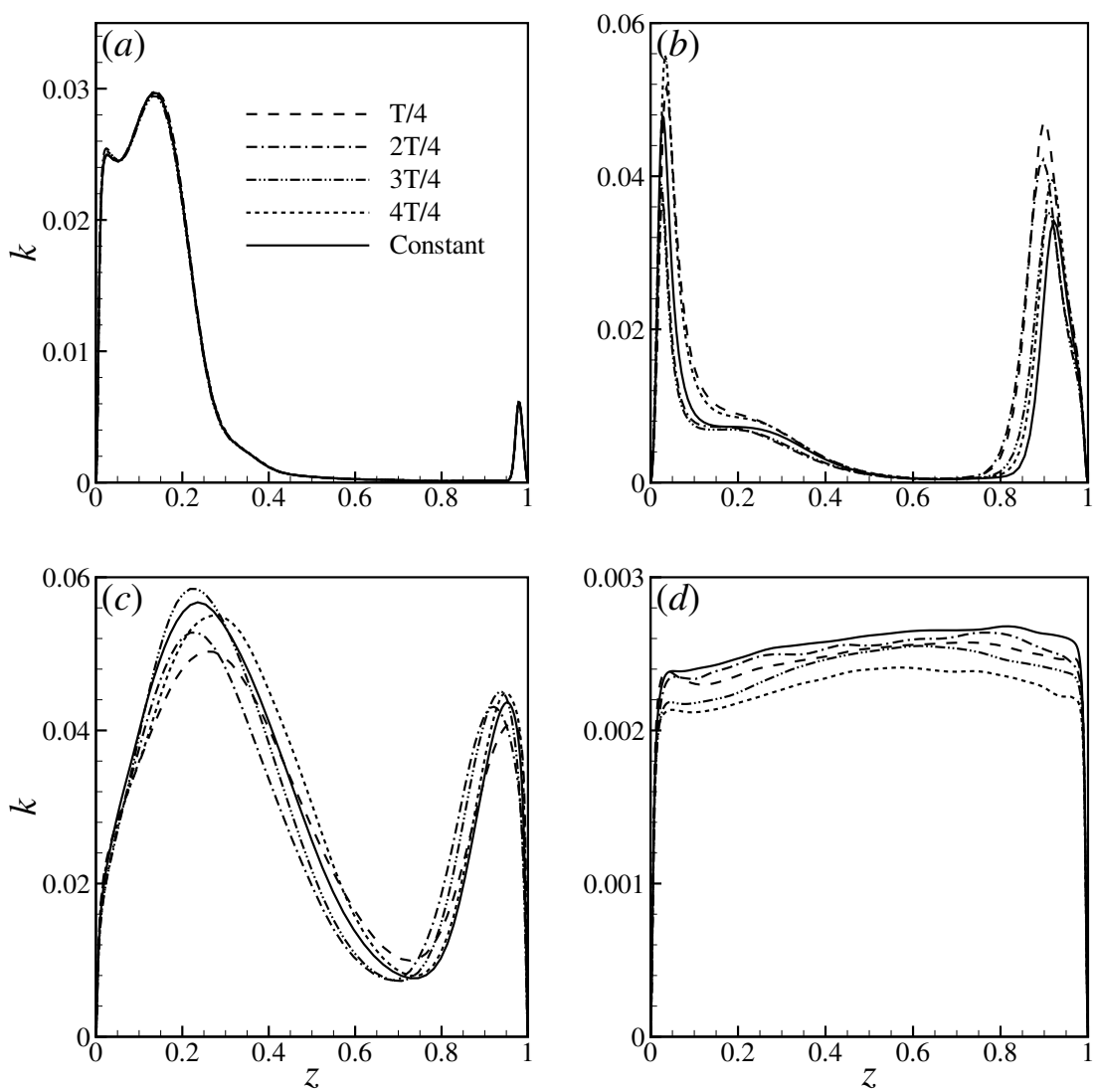

Figure 9: The vertical distributions of the phase-averaged TKE for high frequency backpressure at several streamwise locations $x=$ (a) 1.75 , (b) 5 , (c) 7.5 and (d) 18, respectively. The solid line denotes the TKE for the constant backpressure.

\subsubsection{Turbulent shear stress}

As the oscillatory backpressure has an important influence on the evolution of shear layer, the relevant features are further investigated. Fig. 10 shows the profiles of local turbulent shear stress $\tau_{x z}^{\prime \prime}=\left\{u^{\prime \prime}{ }_{x} u^{\prime \prime}{ }_{z}\right\} / U_{\infty}^{2}$ along the shear layers near the walls, where the location of shear layer is determined in terms of local peak magnitude of shear stress [21, 34,35].

For the low frequency backpressure, the shear stress along the shear layer near the upper wall in Fig. 10(a) exhibits an obvious change at different phases. At $2 \mathrm{~T} / 4$, the turbulent shear stress first experiences an increase around $x=2.7$ due to strong flow separation. At $T / 4$ and 3T/4, the flow separation evolves downstream, resulting in a delay position for the increase of turbulent shear stress. The small flow separation at $4 T / 4$ leads to a small peak of the shear stress. Then in the downstream of the SSS, the shear stress has a quick decrease and even becomes negative. At $2 T / 4$, the shear stress experiences a 

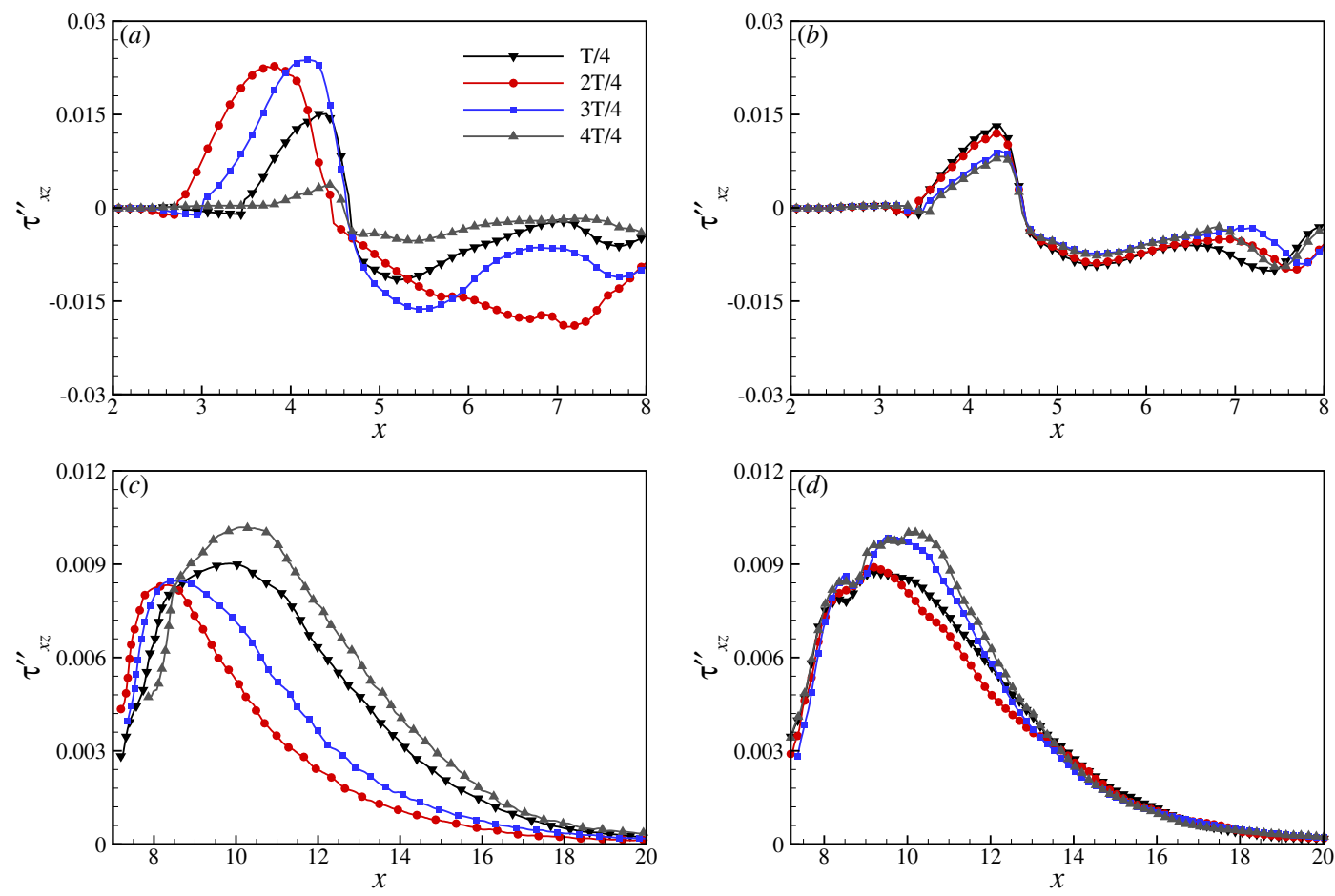

Figure 10: Distributions of the phase-averaged turbulent shear stress along the shear layer near the upper wall for (a) low and (b) high frequency backpressure, and along the shear layer near the lower wall for (c) low and (d) high frequency backpressure.

gradual decrease and reaches its lowest value around $x=7$. With the suppression of flow separation at $T / 4$ and $3 T / 4$, the separation reattachment occurs and then leads to a sharp variation of the shear stress. Then the reattached boundary layer separates again due to high adverse pressure gradient, which results in an increase of the shear stress as well. Moreover, the pressure gradient is the smallest at $4 T / 4$, thus the shear stress has a slight change. On the other hand, for the high frequency backpressure, as shown in Fig. 10(b), the profiles of the shear stress have the similar tendency to those in Fig. 10(a) with small differences among different phases. The shear stress has larger peak value and more forward position of the peak at $T / 4$ and $2 T / 4$ for $x<7$, which corresponds to the forward expanding of flow separation on the upper wall. For $x>7$, the flow separation reattaches at all phases, which results in the shear stress decreasing first and increasing again.

Because of the oscillation of flow separation on the lower wall for $x>7$, the profiles of the shear stress also exhibit the corresponding change under the influence of the oscillatory backpressure, especially for the low frequency backpressure in Fig. 10(c). Compared to the situation near the upper wall, the shear layer due to the flow separation on the lower wall becomes weaker, which results in small shear stress too. Similar to the separated shear layer on the upper wall, the shear stress on the lower wall for the 
high frequency backpressure has small difference among different phases as shown in Fig. 10(d). As the high frequency backpressure mainly influences the downstream flow field, the higher instantaneous backpressure leads to the stronger separation at $T / 4$ and $2 T / 4$, which further results in the lower peak values of the turbulent shear stress.

\subsubsection{Pressure fluctuation}

The pressure fluctuation is important to understand the flow features in supersonic channel flow with the oscillatory backpressure. To analyze the statistical behaviors of the pressure fluctuation, Fig. 11 shows the probability density functions (PDFs) of the pressure fluctuation $p^{\prime}$ at various phases in the region $5<x<10$. It is seen that the positive pressure fluctuation is favor over the negative one and the positive tails are "fatter" than the negatives ones. Moreover, as shown in Fig. 11(a) for the low frequency backpressure, the PDFs of the pressure fluctuation have obvious difference among different phases. The $\mathrm{PDF}$ at $2 \mathrm{~T} / 4$ has broader range due to the influence of the significant flow separation and shock movement. The PDFs are more skew to the positive pressure fluctuation at $T / 4$ and 3T/4, indicating that both the forward and backward evolutions of flow separation are more inclined to produce positive pressure fluctuation. Moreover, for the high frequency backpressure in Fig. 11(b), the PDFs of the pressure fluctuation exhibit little discrepancy among different phases, and the tendency of the skewness of the PDFs becomes relatively weak.

Fig. 12 shows the streamwise distributions of the phase-averaged rms value of pressure fluctuation, $p_{r m s}^{\prime}$, along the centerline for the oscillatory backpressures. The $p_{r m s}^{\prime}$ for the constant backpressure is also plotted for comparison. As shown in Fig. 12(a), the $p_{r m s}^{\prime}$ for the constant backpressure exhibits several peaks when $x<5$ due to the oscillation of shocks. Further, because of the unsteadiness of SSS and its reflected shock, $p_{r m s}^{\prime}$ increases to a peak value around $x=7$ and then decreases quickly. The shear layer induced by the
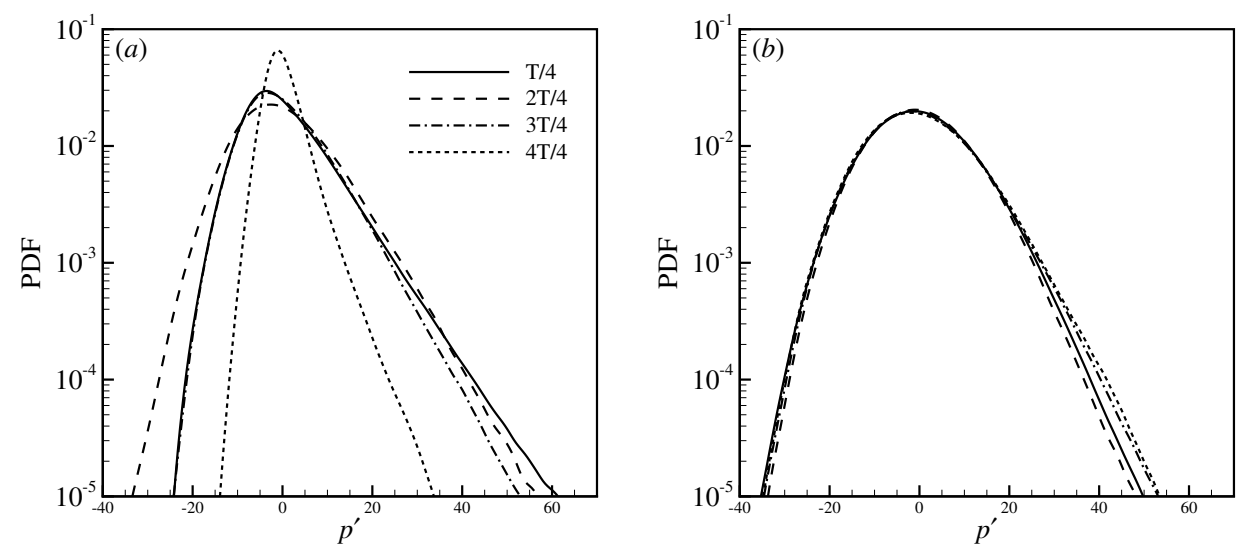

Figure 11: The probability density functions (PDFs) of the pressure fluctuation $p^{\prime}$ at various phases in the region $5<x<10$ for (a) low frequency backpressure and (b) high frequency backpressure. 

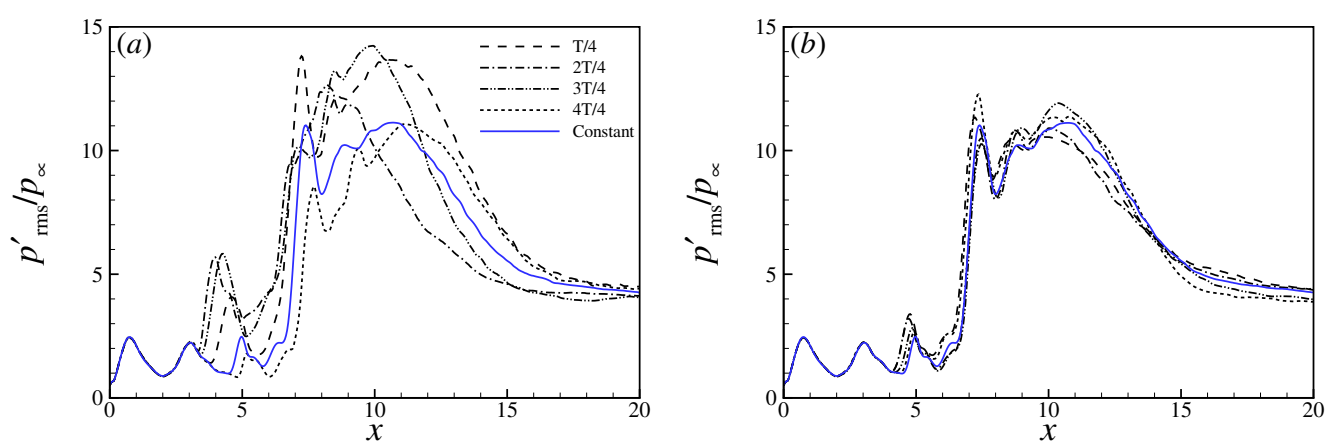

Figure 12: Streamwise distributions of phase-averaged pressure fluctuation $p_{r m s}^{\prime}$ along the centerline for the case with (a) low frequency backpressure and (b) high frequency backpressure. The solid line denotes $p_{r m s}^{\prime}$ for the case with constant backpressure.

flow separation on the lower wall also has a remarkable oscillation around $x=10$, resulting in another peak of $p_{r m s}^{\prime}$. In the downstream of the channel for $x>11$, the pressure fluctuation decreases gradually.

As shown in Fig. 12(a), there exist obvious differences among various phases for the low frequency backpressure. In the upstream of the channel $(x<5)$, the pressure fluctuation at $4 T / 4$ behaves as the constant backpressure case, while the profiles at other three phases are obviously higher. As shown in Fig. 5(a), a small flow separation on the upper wall at $4 T / 4$ results in the pressure fluctuation. At $2 T / 4$, obvious flow separation on the upper wall and the movement of the SSS induce a peak value of $p_{r m s}^{\prime}$ around $x=4$. Meanwhile, the movement of the SSS at $T / 4$ and $3 T / 4$ also leads to higher peak value of the pressure fluctuation. The pressure fluctuation at $T / 4$ and $4 T / 4$ exhibits a peak value around $x=7$. Owing to the reduction of flow separation on the upper wall, the pressure fluctuation at $4 T / 4$ has a peak value around $x=7$ which is lower than that for the constant backpressure. The unsteadiness of the shear layers is also enhanced by the flow separation on the upper wall at $T / 4$, which leads to a higher peak value of the pressure fluctuation as well. In the downstream of the channel $(x>12)$, the pressure fluctuation at several phases tends to collapse together.

The profiles of the phase-averaged pressure fluctuation for the high frequency backpressure are shown in Fig. 12(b). The flow oscillation in the region $x<7$ has an influence on the increase of the pressure fluctuation, especially at $T / 4$ and $2 T / 4$. In the downstream of the channel, the oscillatory backpressure results in higher pressure fluctuation at $T / 4$ and $2 T / 4$ and lower pressure fluctuation at $3 T / 4$ and $4 T / 4$.

\subsection{Unsteady features of the flow field}




\subsubsection{Evolution of flow separation on the wall}

From the preceding discussion, the oscillatory backpressure has an important influence on the flow structures and their unsteady evolution. To obtain a deep insight into the flow physics, the unsteady features of the flow separation are discussed further. Here, the evolution of the flow separation on the upper wall is analyzed. Fig. 13(a) shows the location of the upstream edge of the flow separation zone on the upper wall for the oscillatory backpressure. The upstream edge of the separation zone is located at the position where the skin friction coefficient experiences a zero value and becomes negative in the downstream [36].

The oscillatory motion of the flow separation for the low frequency can be clearly demonstrated in Fig. 13(a). The location of the upstream edge experiences a global variation with a region ranging from $x=2.5$ to 3.8. Moreover, a local high frequency oscillation is also observed from the time-dependent location, which is associated with the backpressure capability. The local oscillation magnitude of the upstream edge at $x=2.5$ is obviously larger than that at $x=3.8$, which is related to the greater adverse pressure gradient due to the flow separation. Furthermore, the upstream edge of the flow separation zone for the high frequency backpressure oscillates ranging from $x=3$ to 3.8 , indicating that the high frequency backpressure has a relative weak influence on the evolution of flow structures in the upstream region. However, the evolution of the flow separation zone is not so obvious under the influence of the high frequency backpressure.

Furthermore, the power spectral densities (PSDs) of the flow separation position are shown in Fig. 13(c). The PDF profiles contain some discrete peaks for the two cases. The PSD has a peak at $S t=0.01$ for the low frequency backpressure and $S t=0.1$ for the high frequency one. These dominant frequencies correspond to the oscillatory backpressure frequencies, which reveals the influence of the oscillatory backpressures on the unsteady features of the flow structures.

On the other hand, the peak value at $S t=0.01$ for the low frequency backpressure is
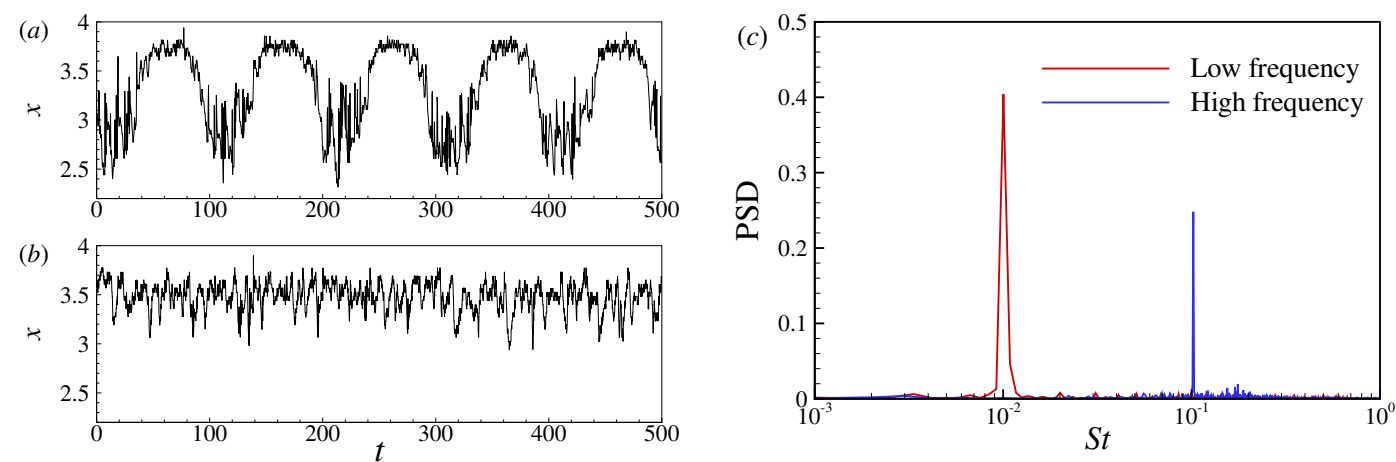

Figure 13: The time history of the position of the upstream edge of flow separation zone on the upper wall for (a) low frequency backpressure and (b) high frequency backpressure; (c) the power spectral densities of the separation position. 
higher than that at $S t=0.1$ for the high frequency one, indicating that the low frequency backpressure has a greater influence on the unsteady evolution of the flow structures with respect to the high frequency backpressure case.

\subsubsection{Energy spectra of the streamwise velocity fluctuation}

The unsteadiness of the flow structures is further investigated by the energy spectra of the velocity fluctuation. Fig. 14 shows the contour of the energy spectra of the streamwise velocity fluctuation as a function of the wave number $m$ and time $t$. The dominant wave number exhibits a periodic-like variation for the low frequency backpressure in Fig. 14(a), while the periodic-like variation is not obvious for the high frequency backpressure in Fig. 14(b). The periodic-like variation of the dominant wave number demonstrates the temporal change scale of flow structures. On the other hand, compared with the location of flow separation in Fig. 13(a), the period with high wave number experiences the high frequency oscillation of the flow separation on the upper wall when its upstream edge tends to $x=2.5$, while the period with low wave number corresponds to the forward and backward movement of the flow separation zone.

The scale change of flow structure will result in significant pressure fluctuation. Hence, the conditional average pressure and pressure fluctuation for high and low wave numbers during a time period are investigated for the low frequency backpressure. The distributions of the conditional average pressure and pressure fluctuation along the centerline for the two typical periods are shown in Fig. 15. Because of the evolution of the flow separation as discussed above, as shown in Fig. 15(a), the conditional average pressure for high wave number achieves a higher value with respect to the mean pressure for the constant backpressure, and a lower value for low wave number. Moreover, the backpressure experiences a reduction in high wave number period, which leads to lower mean pressure near the outlet of the channel for high wave number and higher mean pressure for low wave number.
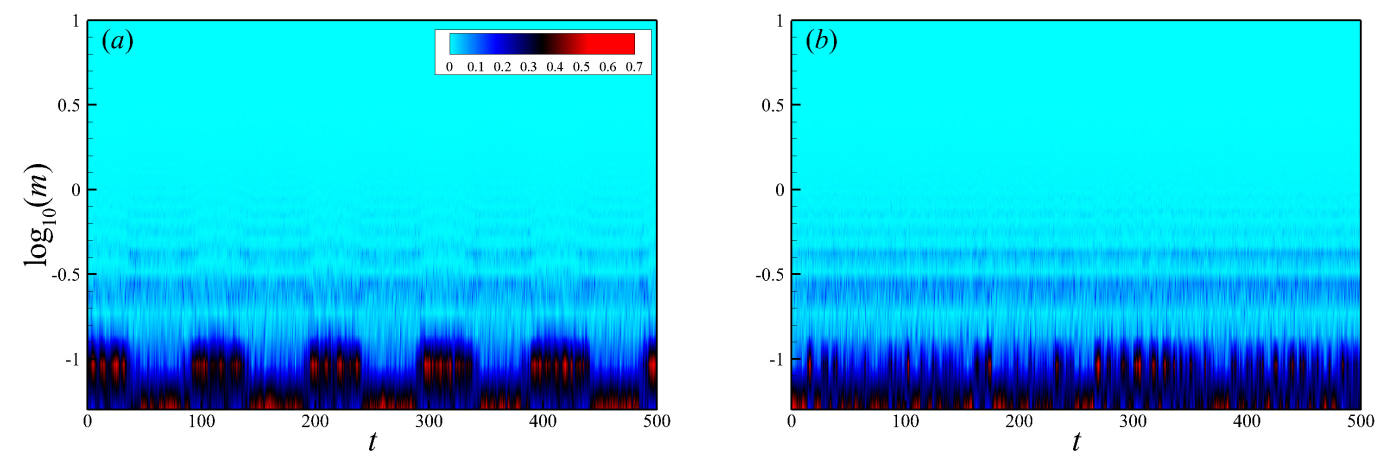

Figure 14: The energy spectra of the streamwise velocity fluctuation as a function of the wave number $m$ and time series for (a) low frequency backpressure and (b) high frequency backpressure. 

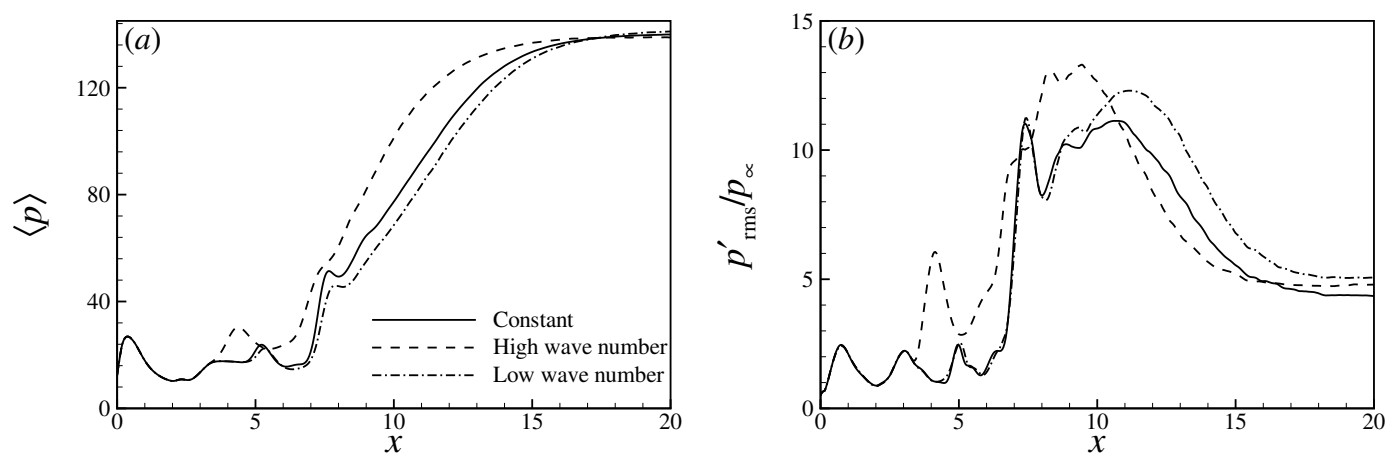

Figure 15: The conditional average (a) pressure and (b) pressure fluctuation for high and low wave number during a time period along the centerline for the low frequency backpressure case. The solid line denotes the mean pressure in (a), and pressure fluctuation in (b) for the constant backpressure case.

The distributions of the conditional average pressure fluctuation are shown in Fig. 15(b). In the upstream of the channel $(x<10)$, the pressure fluctuation for high wave number has relatively higher value in comparison with that for low wave number. In the downstream of the channel, the time period with low wave number also has significant pressure fluctuation under the influence of the flow separation on the lower wall, while the peak value locates around $x=11$. In the near outlet region $(x>17)$, the profiles of the pressure fluctuation for the oscillatory backpressure are higher than that for the constant backpressure.

\subsubsection{The power spectral analysis}

Fig. 16 shows the pressure spectra as a function of the Strouhal number $S t$ for the streamwise location along the centerline of the channel. As shown in Fig. 16(a), the dominant frequency is $S t=0.01$ for the low frequency backpressure. In addition, the dominant frequency of $S t=0.1$ for the high frequency backpressure is also observed in Fig. 16(b), consistent with the oscillatory frequency of backpressure. Similarly, the peak value at $S t=0.1$ in Fig. 16(a) is larger than that at $S t=0.01$ in Fig. 16(b), indicating that the low frequency backpressure has a greater influence on the periodic-like oscillation of flow structures than the high frequency case. Moreover, the pressure spectra for the constant backpressure is shown in Fig. 16(c). The dominant frequency is not obvious as demonstrated in the oscillatory backpressure cases. Nevertheless, a dominant frequency of $S t=0.012$ can be observed around $x=5$, which should be responsible for the local enhancement of the pressure fluctuation.

For a further comparison of the dominant frequency between the low frequency and the constant backpressure, the spatial structures of the low-frequency Fourier mode at $S t=0.01$ are examined in Fig. 17 by means of the treatment [29]. As the oscillation frequency of SSS is approximately $S t=0.01$, the evolution of the SSS has an important influence on the unsteadiness of the flow field. Nevertheless, the energy levels have lower 

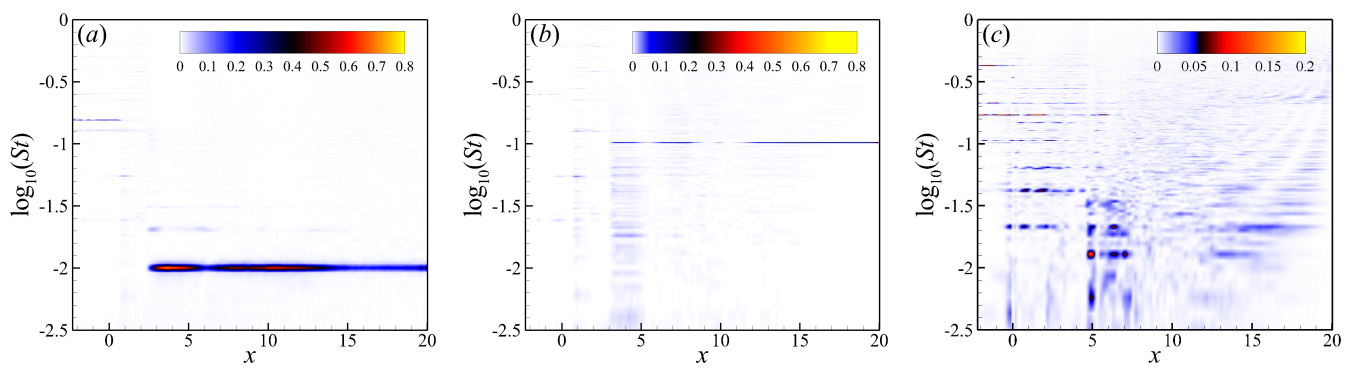

Figure 16: The pressure spectra for (a) the low frequency backpressure, (b) the high frequency backpressure and (c) the constant backpressure.
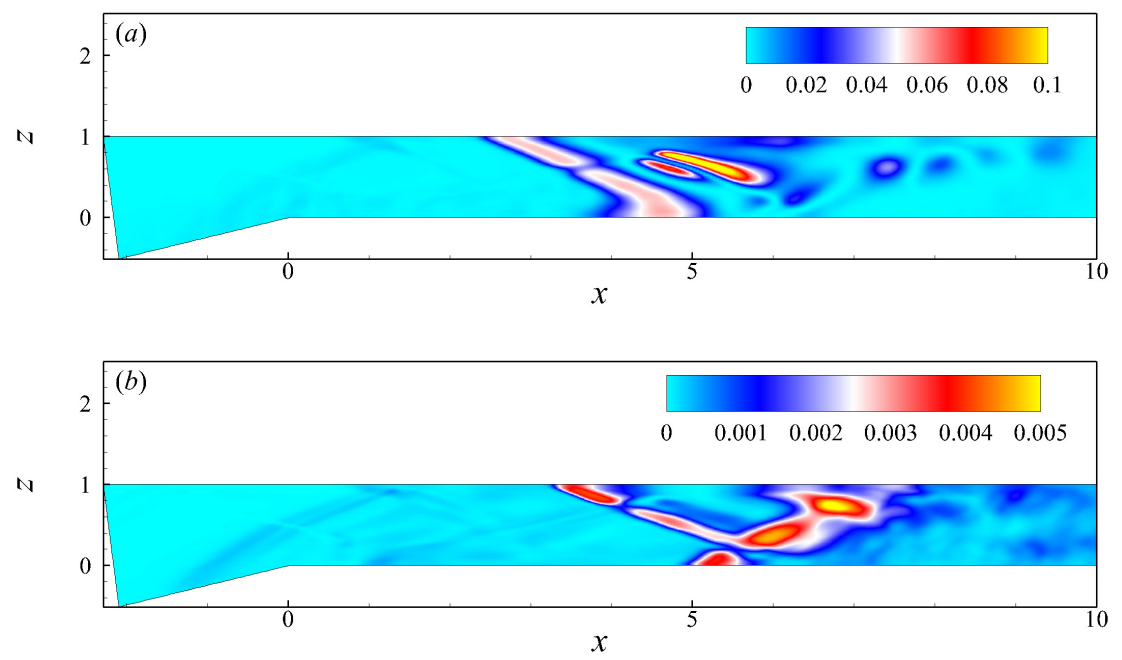

Figure 17: Fourier mode at $S t=0.01$ of the pressure fluctuation for (a) the low frequency backpressure and (b) the constant backpressure.

order for the constant backpressure compared with that for the low frequency backpressure. This implies that the resonance effect of the oscillatory frequency of backpressure and the oscillation frequency of the SSS enhances the unsteadiness of the shock and in turn the pressure fluctuation.

\section{Concluding remarks}

Numerical investigation on the complex flow phenomena and mechanisms in supersonic channel flow with periodic oscillatory backpressures at the outlet of the channel was carried out by means of the LES technique. Three kinds of backpressure for constant backpressure and both oscillatory backpressures with low and high frequency were considered. The statistical features of flow field and pressure fluctuations were examined. Vari- 
ous fundamental mechanisms dictating the complex flow phenomena, including moving shock waves, flow separations, shear layers, were investigated.

It is found that the low frequency backpressure leads to a periodic-like oscillation of flow structures and the enhancement of the flow separation on the upper wall, while the high frequency backpressure relatively has a weak influence on the periodic-like motion of flow structures. The averaged flow structures demonstrate that the similar structures occur for different backpressures. Based on the analyse of the phase-averaged quantities, it is identified that there exists the phase difference of flow structures for the low frequency backpressure. The oscillatory backpressures have a significant influence on the statistical features. Because the oscillatory backpressure is applied, the flow separation on the upper wall is enhanced, which leads to an enhancement of the oscillation of the separation shock. As a consequence, turbulence intensities are also enhanced. In particular, due to the influence of the low frequency backpressure, the phase-averaged turbulent kinetic energy has an obvious phase dependence. Moreover, large pressure fluctuation is observed for the low frequency backpressure at various phases, especially in the middle region of the channel.

The unsteadiness of flow field has been examined by the evolution of flow structures. It is found that the flow structures globally have low frequency oscillation under the influence of the low frequency backpressure. The dominant frequency of the evolution of the flow structures is close to the frequency of the oscillatory backpressure. Moreover, the low frequency backpressure leads to obvious periodic-like evolution of the flow structures. Due to the resonance effect between the low frequency backpressure and the oscillation of the secondary separation shock, the low frequency backpressure results in an enhanced oscillation of the shock and a significant pressure fluctuation in the channel.

\section{Acknowledgements}

This work was supported by the National Natural Science Foundation of China (Nos. 11572312, 11621202, 11472268, and 91752110) and by Science Challenge Project (No. TZ2016001).

\section{References}

[1] D. M. VAN WIE, F. T. KWOK AND R. T. WALSH, Starting characteristics of supersonic inlets, AIAA Paper, (1996), pp. 2914.

[2] J. L. WagneR, K. B. Yuceil, A. Valdivia, N. T. Clements And D. S. Dolling, Experimental investigation of unstart in an inlet/isolator model in Mach 5 flow, AIAA J., 47 (2009), pp. 1528-1542.

[3] E. T. CURran, W. H. Heiser AND D. T. PrATt, Fluid phenomena in scramjet combustion systems, Annu. Rev. Fluid Mech., 28 (1996), pp. 323-360.

[4] W. H. HEISER AND D. T. PRATT, Hypersonic air breathing propulsion, AIAA Education Series, AIAA, (1993). 
[5] H. KOO AND V. RAMAN, Large-eddy simulation of a supersonic inlet-isolator, AIAA J., 50 (2012), pp. 1596-1613.

[6] Z.-F. LI, W.-Z. GAO, H.-L. JIANG AND J.-M. YANG, Unsteady behaviors of a hypersonic inlet caused by throttling in shock tunnel, AIAA J., 51 (2013), pp. 2485-2492.

[7] J. L. WAGNER, K. B. YUCEIL AND N. T. CLEMENTS, Velocimetry measurements of unstart in an inlet-isolator model in Mach 5 flow, AIAA J., 48 (2010), pp. 1875-1888.

[8] H.-J. TAN AND S. SUN, Oscillatory flows of rectangular hypersonic inlet unstart caused by downstream mass-flow choking, J. Propul. Power, 25 (2009), pp. 138-147.

[9] A. INGENITO, C. BRUNO AND D. CECERE, LES of the hyshot scramjet combustor, AIAA Paper, (2010), 758.

[10] P. Cocks, J. Donohue, C. BRUnO AND M. HAAS, IDDES of a dual-mode ethylene fueled cavity flameholder with an isolator shock train, AIAA Paper, (2013), 0116.

[11] L. KRISHNAN, N. D. SANDHAM AND J. STEELANT, Shock-wave/boundary-layer interactions in a model scramjet intake, AIAA J., 47 (2009), pp. 1680-1691.

[12] R. BUR, R. BENAY, A. GALli AND P. BERTHOUZE, Experimental and numerical study of forced shock-wave oscillations in a transonic channel, Aerosp. Sci. Technol., 10(4) (2006), pp. 265-278.

[13] P. J. K. BRUCE AND H. BABINSKY, Unsteady shock wave dynamics, J. Fluid Mech., 603 (2008), pp. 463-473.

[14] C. Cheng, C.-P. WANG, K.-M. Cheng AND J. YUn, Experimental study of unsteady oblique shock train, AIAA Paper, (2017), 2104.

[15] W.-Y. SU AND K.-Y. ZHANG, Back-pressure effects on the hypersonic inlet-isolator pseudoshock motions, J. Propul. Power, 29 (2013), pp. 1-9.

[16] K.-J. XU, J.-T. CHANG, W.-X. ZHOU AND D.-R. YU, Mechanism and prediction for occurrence of shock-train sharp forward movement, AIAA J., 54 (2016), pp. 1403-1412.

[17] S. TRAPIER, S. DECK AND P. DUVEAU, Delayed detached-eddy simulation and analysis of supersonic inlet buzz, AIAA J., 46 (2008), pp. 118-131.

[18] J.-T. CHANG, N. LI, K.-J. XU, W. BAO AND D.-R. YU, Recent research progress on unstart mechanism, detection and control of hypersonic inlet, Prog. Aerosp. Sci., 89 (2017), pp. 1-22.

[19] X.-Y. LU, S.-W. WANG, S.-W. SUNG, S. Y. HsiEH AND V. YANG, Large-eddy simulations of turbulent swirling flows injected into a dump chamber, J. Fluid Mech., 527 (2005), pp. 171-195.

[20] C.-Y. XU, L.-W. CHEN AND X.-Y. LU, Large-eddy simulation of the compressible flow past a wavy cylinder, J. Fluid Mech., 665 (2010), pp. 238-273.

[21] L.-W. CHEN, G.-L. WANG AND X.-Y. LU, Numerical investigation of a jet from a blunt body opposing a supersonic flow, J. Fluid Mech., 684 (2011), pp. 85-110.

[22] N. D. SANDHAM, Y.-F. YAO AND A. A. LAWAL, Large-eddy simulation of transonic turbulent flow over a bump, Int. J. Heat Fluid Flow, 524 (2003), pp. 584-595.

[23] Q. LI AND G. N. COLEMAN, DNS of an oblique shock wave impinging upon a turbulent boundary layer, Direct and Large-Eddy Simulation V, 9 (2003), pp. 387-396.

[24] S. PIROZZOLI, M. BERNARDINI AND F. GRASSO, Characterization of coherent vortical structures in a supersonic turbulent boundary layer, J. Fluids Mech., 613 (2008), pp. 205-231.

[25] S. Pirozzoli, M. Bernardini AND F. GRASSO, Direct numerical simulation of transonic shock/boundary layer interaction under conditions of incipient separation, J. Fluids Mech., 657 (2010), pp. 361-393.

[26] L. WANG AND X.-Y. LU, Flow topology in compressible turbulent boundary layer, J. Fluids Mech., 703 (2012), pp. 255-278.

[27] S. PiROzZOLI, M. BERNARDINI AND F. GRASSO, On the dynamical relevance of coherent vortical structures in turbulent boundary layer, J. Fluids Mech., 648 (2010), pp. 325-349. 
[28] N. T. Clemens AND V. NARAYANASWAMY, Low-frequency unsteadiness of shock wave/turbulent boundary layer interactions, Annu. Rev. Fluid Mech., 46 (2014), pp. 469-492.

[29] F. SARTOR, C. MetTOT, R. BUR AND D. SipP, Unsteadiness in transonic shock-wave/boundarylayer interactions: experimental investigation and global stability analysis, J. Fluids Mech., 781 (2015), pp. 550-577.

[30] G. Matheou, A. M. Bonanos, C. Pantano and P. E. Dimotakis, Large-eddy simulation of mixing in a recirculating shear flow, J. Fluids Mech., 646 (2010), pp. 375-414.

[31] L.-W. CHEN, C.-Y. XU AND X.-Y. LU, Numerical investigation of the compressible flow past an aerofoil, J. Fluid Mech., 643 (2010), pp. 97-126.

[32] M. DiNG, L. HuA AND F. XIAO, Two-dimensional viscous simulation of inlet-isolator flows at Mach number 4, AIAA Paper, (2001), 3883.

[33] C. BOGEY AND C. BAILlY, Influence of nozzle-exit boundary-layer conditions on the flow and acoustic fields of initially laminar jets, J. Fluids Mech., 663 (2010), pp. 507-538.

[34] J. L. HERRIN AND J. C. DUTTON, The turbulence structure of a reattaching axisymmetric compressible free shear layer, Phys. Fluids, 9 (1997), pp. 3502-3512.

[35] F. Simon, S. DecK, P. Guillen, P. SAgaut And A. Merlen, Numerical simulation of the compressible mixing layer past an axisymmetric trailing edge, J. Fluids Mech., 591 (2007), pp. 215-253.

[36] B. Morgan, K. Duraisamy, N. Nguyen, S. Kawai and S. K. Lele, Flow physics and RANS modelling of oblique shock/turbulent boundary layer interaction, J. Fluids Mech., 729 (2013), pp. 231-284. 